\title{
NECROSIS DE PENE: REVISIÓN DE 18 CASOS EN EL HOSPITAL DE ESPECIALIDADES CENTRO MÉDICO NACIONAL SIGLO XXI.
}

\author{
Guillermo Montoya Martínez, José Manuel Otero García, Virgilio López Samano, José \\ González Martínez y Eduardo Serrano Brambila.
}

Servicio de Urología del Hospital de Especialidades Centro Medico Nacional Siglo XXI, México Distrito Federal, México.

\begin{abstract}
Resumen.- OBJETIVO: Presentar la experiencia en el manejo de necrosis de pene en nuestro centro hospitalario.

MÉTODOS: Se realizó la revisión de expedientes clínicos con diagnóstico de necrosis de pene en el servicio de Urología del Hospital de Especialidades Centro Medico Nacional Siglo XXI de enero de 1995 a marzo de 2005.
\end{abstract}

RESULTADOS: Se encontraron 18 casos con necrosis de pene con edades entre 28 a 78 años ledad media de 58.1 años). Los antecedentes personales patológicos con mayor incidencia fueron la diabetes mellitus en 10 casos e insuficiencia renal crónica terminal en 11 casos. Los motivos de consultas fueron presencia de escaras en pene en cinco casos, exudado purulento,

José Manuel Otero García

Servicio de Urología Hospital de Especialidades. 3er Piso Centro Medico Nacional Siglo XXI

Avenida Cuauhtémoc \# 330 Colonia Doctores Delegación Cuauhtémoc 06720, México Distrito Federal (México). drimanuelog@hotmail.com

Trabajo recibido: 29 de julio 2005 inflamación de glande y prepucio en tres casos, priapismo isquémico en dos casos, síndrome de Fournier dos casos, uretrorrea un caso, necrosis de pene secundario a compresión extrínseca en un caso y absceso periuretral un caso. De los 78 casos a 13 se les realizó penectomía total o parcial, de los cuales 3 fallecieron. A 5 pacientes se les dio manejo conservador con antibióticos, uno de ellos falleció. El análisis de patología reportó trombosis arterial y venosa además de necrosis isquémica en 3 casos; trombosis arterial y venosa, necrosis isquémica y calcificación distrófica arterial y venosa en 10 casos.

CONCLUSIONES: Los cambios vasculares oclusivos son un factor condicionante en la mayoría de los pacientes con necrosis de pene, esto además se hace más evidente en pacientes con insuficiencia renal crónica terminal, diabetes mellitus e hipertensión arterial sistémica. Las manifestaciones clínicas de la necrosis de pene son escaras, momificación, autoamputación y sobreinfección, es por eso que el diagnostico temprano y el tratamiento oportuno es determinante en la evolución de este padecimiento.

Palabras clave: Necrosis de pene. Manifestaciones clínicas. Manejo.

Summary.- OBJECTIVES: To report the experience in the management of penile necrosis at our hospital.

METHODS: We performed a review of the medical records with the diagnosis of penile necrosis at the Department of the Urology of the Hospital de Especialidades Centro Médico nacional Siglo XXI from January 1995 to March 2005.

RESULTS: 18 cases of penile necrosis were found, with ages from 28 to 78 years (mean age 58.1 yr.). Diabetes 
mellitus and end stage renal disease were the most frequent associated diseases, with 10 and 11 cases respectively. Reasons for consultation were penile scars in 5 cases, purulent exudation, inflammation of the glans penis and prepuce in 3 , ischemic priapism in 2 , Fournier's syndrome in 2, urethral bleeding in one , penile necrosis secondary to extrinsic compression in one, and periurethral abscess in another. Thirteen out of the 18 cases underwent partial or complete penectomy, and three of them died. Five patients were managed conservatively with antibiotics, one of them died. The pathology report showed ischemic necrosis in 3 cases, arterial and venous thrombosis, ischemic necrosis and dystrophic arterial and venous calcifications in 10 cases.

CONCLUSIONS: Occlusive vascular changes are a conditioning factor in most patients with penile necrosis. This is more evident in patients with end stage renal disease, diabetes mellitus and hypertension. Clinical features of penile necrosis include scars, mummification, self-amputation, and superinfection, so that an early diagnosis and proper treatment are decisive for the evolution of this disease.

Keywords: Penile necrosis. Clinical features.

Management.

\section{OBJETIVO}

Presentar la experiencia en el manejo de la necrosis de pene en nuestro centro y compararla con la experiencia publicada en la literatura.

\section{INTRODUCCIÓN}

La necrosis del pene ha sido descrita en casos de pacientes con diabetes mellitus descontrolada, posterior a colocación de prótesis de pene y secundaria a depósitos intravasculares de calcio en pacientes con diálisis crónica (1-3). También se ha encontrada asociada a gangrena de Fournier, compresión externa de pene con efecto de torniquete, administración de warfarina, pioderma gangrenoso, herpes simple tipo I (4), púrpura trombocitopénica idiopática (5), y priapismo causado por drogas como la cocaína (6).

El diagnóstico se hace clínicamente, por los antecedentes, la exploración física y estudios de gabinete que orientan hacia la naturaleza del padecimiento. $(1,4,7)$.

Las opciones de tratamiento incluyen la conducta conservadora (observación, antibióticos, folatos, oxigeno hiperbárico) y el tratamiento quirúr- gico (penectomía parcial o total con o sin derivación urinaria por cistostomía). $(4,7)$. Se ha observado de manera constante un mal pronóstico en pacientes con necrosis de pene e Insuficiencia renal crónica terminal (IRCT).

La experiencia en el manejo de este padecimiento es limitada; se han publicado a la fecha menos de cuarenta casos en la literatura mundial, la serie más grande reporta 7 casos.

\section{MATERIALES Y MÉTODOS}

Se revisaron los expedientes clínicos de los pacientes con diagnóstico de necrosis de pene en el Servicio de Urología del Hospital de Especialidades Centro Medico Nacional Siglo XXI de Enero de 1995 a Marzo de 2005.

\section{RESULTADOS}

Se encontraron un total de 18 casos, la edad media fue de 58.1 años de edad con un rango de 28 a 78 años de edad. De los expedientes revisados encontramos los siguientes antecedentes: tabaquismo en un paciente, alcoholismo y tabaquismo concomitante en otro y un paciente con adicción a cocaína, 5 pacientes con IRCT secundario a diabetes mellitus tipo II; 2 pacientes con IRCT secundaria a hipertensión arterial sistémica y diabetes mellitus tipo II, uno más con IRCT secundaria a hipertensión arterial sistémica, tres pacientes con IRCT secundaria a glomerulonefritis, uno con diabetes mellitus tipo II sola, uno con hipertensión arterial sistémica con tratamiento irregular y uno con diagnóstico de SIDA en etapa C3 (Tabla I). De los casos con IRCT 5 eran tratados mediante hemodiálisis, 4 diálisis peritoneal continua ambulatoria, uno con tratamiento medico y el restante con trasplante de donador vivo, manejado con inmunosupresores (micofelonato, prednisona y rapamicina).

La causa más frecuente de consulta fue la presencia de exudado purulento e inflamación en glande y prepucio. Esto lo observamos en seis de los casos (33.3\%), con edades de 39 a 65 años de edad. Tres de ellos (16.6\%) evolucionaron a necrosis total de pene en un periodo de una semana y media a 6 semanas y ameritaron penectomía total con meato perineal. En dos de los casos (11.1\%) no hubo progresión de la necrosis del glande y se les menejó con penectomía parcial. El último caso $(5.5 \%)$ de 52 años de edad tuvo autoamputación progresiva del pene hasta la unión penoescrotal en un periodo de seis meses hasta que solicitó atención médica rea- 
lizándose neopene con colgajo musculocutáneo de antebrazo y neouretra con piel de abdomen. Todos ellos vivos hasta el momento.

La segunda causa de consulta fue la presencia de una escara en prepucio y/o glande, en cinco casos. En tres pacientes $(16.6 \%)$ solo involucró al prepucio y fueron sometidos a manejo conservador con antibióticos, tuvieron adecuada epitelización posterior a las tres semanas en promedio, solo con perdida distal de glande y prepucio. Los otros dos pacientes $(11.1 \%)$ evolucionaron a necrosis total de pene y sepsis. Un caso $(5.5 \%)$ ameritó penectomía total y el otro se manejó conservadoramente con antibióticos, ambos fallecieron a las 2 y 6 semanas respectivamente por complicaciones infecciosas (Figura 1).

Dos pacientes (11.1\%) acudieron por priapismo isquémico. El primero de 48 años de edad con priapismo secundario al consumo de cocaína, quien se presentó a las 83 horas después del inicio y remitió con la irrigación de cuerpos cavernosos. Evolucionó a Síndrome de Fournier con necrosis húmeda total del pene a los cuatro días posteriores al procedimiento. El segundo caso de 63 años de edad se presentó con priapismo primario de 72 horas de evolución, se sometió a irrigación de cuerpos cavernosos, remitiendo el priapismo totalmente. Presentó necrosis total de pene a las 96 horas del postoperatorio. En ambos casos se requirió penectomía total; el primero se le realizó neopene con colgajo musculocutáneo de gracilis y el segundo con cistostomía suprapubica y no se observaron complicaciones posteriores.

Un caso $(5.5 \%)$ de 62 años de edad presentó uretrorrea a la semana de colocar sonda transuretral para cuantificación de uresis durante su internamiento por insuficiencia renal crónica. Evolucionó a necrosis húmeda de pene en toda su extensión a los
10 días de colocada la sonda. Ameritó penectomía total con meato perineal y falleció una semana después por acidosis metabólica y estado hiperosmolar. En otro paciente $(5.5 \%)$ de 65 años de edad, con antecedentes de esquizofrenia, se autocolocó un torniquete de alambre metálico en la base del pene por 72 horas. Progresó a necrosis de pene. Se realizó penectomía total con cistostomía suprapubica y no tuvo complicaciones posteriores. Actualmente se encuentra en seguimiento por el servicio de psiquiatría.

Un paciente $(5.5 \%)$ de 78 años de edad diabético y con IRCT, presentó absceso periuretral 10 días después de resección transuretral de próstata, evolucionó a necrosis húmeda de tercio distal de pene. Se realizó penectomía parcial y cistostomía suprapubica. El paciente se perdió del seguimiento por cambio de domicilio.

Finalmente dos pacientes ( $11.1 \%)$ de uno de 51 años de edad, con antecedente de IRCT manejada con trasplante renal de 6 años y el otro de 68 años de edad, ambos ingresaron por Síndrome de Fournier que evolucionó a necrosis de pene a las 8 semanas el primero y a los 9 días el segundo, los dos ameritaron penectomía total y cistostomía. Un paciente falleció por sepsis a los 6 días de la penectomía, el otro en seguimiento por el servicio de trasplantes (Tabla II y III).

Es interesante mencionar que en las radiografías de pelvis se identificaron calcificaciones vasculares de los vasos iliacos y sus ramas principales en 10 de los pacientes con IRCT terminal (excepto en el trasplantado) (Figura 2).

De los dieciocho casos presentados, 13 $(72.2 \%)$ se sometieron a penectomía parcial o total. En el estudio histopatológico se documentó la presencia de necrosis isquémica secundaria a trombosis de

\section{TABLA I.}

\begin{tabular}{|l|c|c|}
\hline \multicolumn{1}{|c|}{ Antecedentes personales } & Numero de casos & $\%$ casos \\
\hline Sin antecedentes personales patológicos de importancia para el padecimiento & 3 & 16.6 \\
\hline Diabetes mellitus & 10 & 55.5 \\
\hline Insuficiencia Renal Crónica Terminal & 11 & 61.1 \\
\hline Hipertensión Arterial Sistémica & 4 & 22.2 \\
\hline SIDA & 1 & 5.5 \\
\hline
\end{tabular}




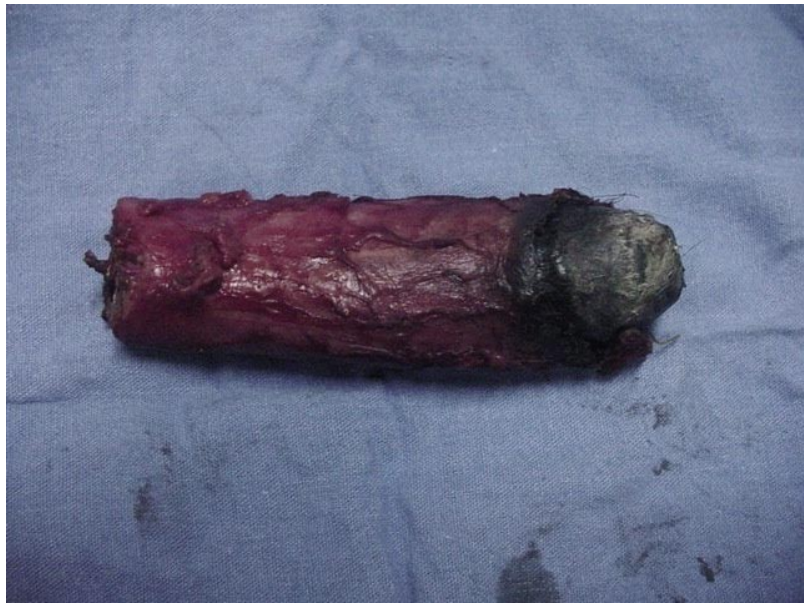

FIGURA 1. Necrosis total de pene.

arterias y venas de mediano y pequeño calibre en tres (16.6\%) especímenes, en diez (55.5\%), trombosis arterial y venosa secundaria a calcificación distrófica de arterias y venas de pequeño y mediano calibre, estos últimos de pacientes con IRCT en tratamiento sustitutivo (Tabla IV y Figura 3).

\section{DISCUSIÓN}

La necrosis de pene es un padecimiento urológico poco común. En la literatura se ha descrito junto con gangrena de Fournier, estrangulación externa, en la administración de warfarina, pioderma gangrenoso, herpes virus tipo I, púrpura trombocitopenica idiopática, diabetes mellitus de larga evolución, insuficiencia renal crónica terminal, enfermedad de Crohn, cirrosis hepática, lupus eritematoso sistémico,

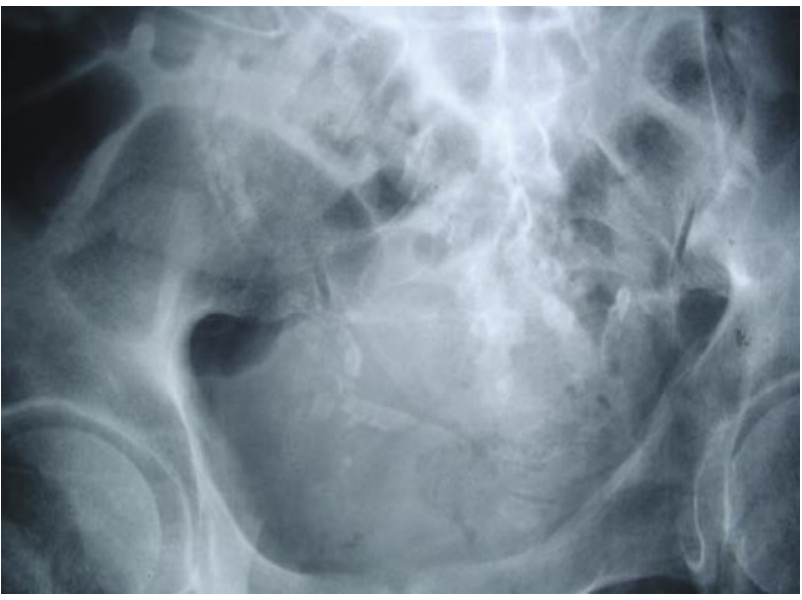

FIGURA 2. Radiografía con calcificaciones vasculares de los vasos iliacos y sus ramas principales.

traumatismos de pene e hipertensión arterial sistémica $(1,4,7,8)$.

Esta es una entidad rara, se han descrito menos de 40 casos en la literatura mundial. En 1974 Rao, Heckman y Olsson reportaron un paciente con gangrena de pene asociado a trombocitopenia; en 1984 Bour y Steinhard reportaron dos pacientes con gangrena de pene asociado a enfermedad renal terminal y diabetes mellitus, microscópicamente encontraron calcificación intravascular de las arterias de mediano calibre $(4,5)$; Ashouri y Pérez en 1986 reportaron un caso de necrosis de pene en el que radiograficamente demostraron calcificaciones vasculares pélvicas $(1,3,4,7,11)$.

Las calcificaciónes intravasculares en pacientes con IRCT terminal, son una alteración producto del

\section{TABLA II.}

\begin{tabular}{|l|c|c|}
\hline \multicolumn{1}{|c|}{ Sintomas iniciales } & Numero de casos & $\%$ casos \\
\hline Escara en pene & 5 & 27.7 \\
\hline Exudado purulento, inflamación de glande y prepucio & 6 & 33.3 \\
\hline Priapismo & 2 & 11.1 \\
\hline Uretrorrea & 1 & 5.5 \\
\hline Compresión extrínseca efecto de torniquete & 1 & 5.5 \\
\hline Absceso periuretral & 1 & 5.5 \\
\hline Gangrena de Fournier & 2 & 11.1 \\
\hline
\end{tabular}


TABLA III.

\begin{tabular}{|l|c|c|c|c|c|c|}
\hline & Tratamiento conservador & $\%$ & Penectomía total & $\%$ & Penectomía parcial & $\%$ \\
\hline Fallecidos & 1 & 5.5 & 3 & 16.6 & 0 & 0 \\
\hline Vivos & 4 & 22.2 & 7 & 38.8 & 2 & 11.1 \\
\hline Sin seguimiento & 0 & 0 & 0 & 0 & 1 & 5.5 \\
\hline
\end{tabular}

TABLA N.

\begin{tabular}{|lcc|}
\hline \multicolumn{1}{|c|}{ Histopatología } & Numero de casos & \% casos \\
\hline Trombosis y necrosis & 3 & 16.6 \\
\hline Trombosis, necrosis y calcificaciones & 10 & 55.5 \\
\hline No hubo muestra patológica & 5 & 27.7 \\
\hline
\end{tabular}

hiperparatiroidismo secundario a hiperfosfatemia. La disminución del filtrado glomerular causa retención de fósforo, estimulando el desarrollo de hiperparatiroidismo por dos mecanismos: favorece la hipocalcemia la cual estimula la secreción y síntesis de la hormona paratiroidea y aumenta la concentración de fósforo directamente sobre las células paratiroideas estimulando la secreción y síntesis de la hormona paratiroidea, la hormona es reponsable de estimular la absorción de calcio a nivel intestinal, la reabsorción renal de calcio. Este estado, condiciona depósito de calcio en los tejidos (calcifilaxis) y se presenta cuando la función renal es menor del $25 \%(11,12)$.

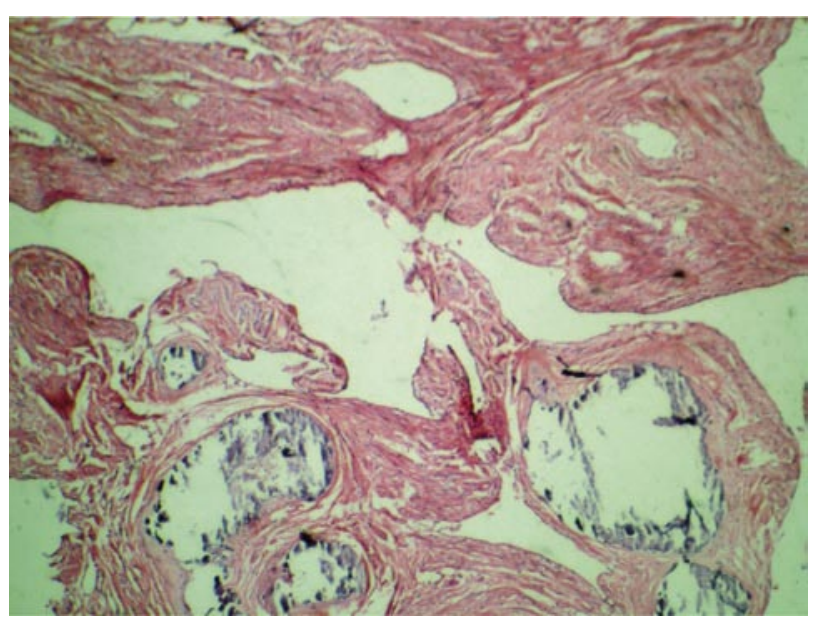

FIGURA 3. Senos cavernosos con necrosis isquémica y calcificación distrófica (x 10)
La calcifilaxis es una condición sistémica poco común, se observa en el $1-4 \%$ de los pacientes con IRCT terminal en tratamiento sustitutivo. La presentación clínica consiste en manchas rojizas en la piel y lesiones violáceas dolorosas en la extremidades dístales, abdomen, nalgas y mamas, evolucionan a necrosis, gangrena y autoamputación (11).

Otro factor etiológico conocido para la necrosis de pene es el consumo de cocaína. Esta inhibe la recaptura de norepinefrina por bloqueo del transporte presináptico y favorece la aparición de priapismo, el cual, generalmente es difícil de tratar y en ocasiones deriva en complicaciones isquémicas irreversibles (6).

En cuanto al cuadro clínico la mayoría de los casos se describen en la literatura como eritema en el pene, una lesión necrótica en el glande, progresiva, que puede involucrar al meato uretral y obstruirlo. Además de dolor en el pene, balanitis o fimosis, uretrorrea, fiebre y acartonamiento del pene $(5,8,12)$. En nuestra casuistica la forma más común de manifestarse esta entidad fue en forma de escara en el glande $y / o$ prepucio.

El tratamiento de la necrosis de pene por calcifilaxis ha consistido en normalizar el calcio sérico y niveles de fosfato, en ocasiones paratiroidectomía (en pacientes con IRCT que cursen con incremento de la paratohormona y calcifilaxis), control de la infección mediante desbridamiento de las áreas necróticas, antibióticos y oxigeno hiperbarico en algunos hospitales $(4,11)$. Steint y colaboradores recomienda 
el tratamiento conservador, sin embargo estos pacientes progresan a momificación y autoamputación en corto tiempo $(7,9)$. Weiner y colaboradores sugieren el manejo quirúrgico temprano ya que puede mejorar la calidad de vida del paciente disminuyendo el dolor por isquemia. Los pacientes que se someten a tratamiento conservador ocasionalmente evolucionan hacia la infección y licuefacción tisular, requiriendo catéteres suprapubicos y/o cirugía (penectomía). También es sabido que la necrosis del glande puede causar obstrucción urinaria (7). Barthelmes por su parte recomienda la cirugía, desbridamiento, emasculación y uretrostomía perineal tempranos, para evitar la superinfección y gangrena (13).

Se ha descrito una alta mortalidad cuando la necrosis de pene se presenta en personas con diabetes mellitus e insuficiencia renal en mas del 50\% a 6 meses $(7,14)$. La diabetes mellitus, hipertensión arterial sistémica, insuficiencia renal crónica y dislipidemia aceleran el proceso de angiopatía ateroesclerótica, constituyendo todos factores de riesgo importantes para esta entidad (4). En nuestra revisión encontramos que 11 pacientes contaban con más de una patología crónico-degenerativa.

\section{CONCLUSIONES}

La necrosis de pene es una entidad clínica secundaria a múltiples patologías que afectan la circulación del pene causando isquemia, necrosis y secundariamente infección en muchos de los casos.

El cuadro clínico de esta entidad son datos inflamatorios, aparición de exudado purulento, escaras, momificación, dolor e incluso la autoamputación del pene. Es por esto que el reconocimiento temprano $y$ tratamiento oportuno de esta enfermedad son determinantes en la evolución de estos pacientes. Dejada a libre evolución progresa a licuefacción, sobreinfección y obstrucción urinaria.

El pronóstico de estos pacientes depende de las enfermedades concomitantes, sin embargo la sobrevida es corta cuando se asocia a insuficiencia renal crónica terminal.

Es necesario reconocer la trascendencia de esta entidad, si bien son raros los reportes encontrados en la literatura, nosotros en nuestro servicio lo encontramos con cierta frecuencia. Lo que nos anima a compartir nuestra experiencia y a buscar pautas del diagnostico temprano y tratamiento oportuno.

\section{BIBLIOGRAFIA y LECTURAS RECOMENDADAS ( ${ }^{*}$ lectura de interés $y^{* *}$ lectura fundamental)}

1. HARRIS, C.F.; MYDLO, J.H.: "Ischemia and gangrene of the penis". J. Urol., 169: 1795, 2003.

2. KARDAR, A.; PETTERSON, B.A.: "Penile gangrene: a complication of penile prosthesis". Scand. Journal. Urol. Nephrol., 29: 355, 1995.

3. HANDA, S.P.; STRZELCZAK, D.: "Uremic small artery disease: calciphylaxis with penis involvement". Clin. Nephrol., 50: 258, 1998.

4. BOUR, J.; STEINHARDT, G.: "Penile necrosis in patients with diabetes mellitus and end stage renal disease". J. Urol., 132: 560, 1984.

5. RAO, C.N.; HECKMAN, H.; OLSSON C.A.: "Trombocytopenia with external genital gangrene”. J. Urol., 122: 208, 1974.

6. ALTMAN, A.; SEFTEL, A.D.; BROWN, S.L. y cols.: "Cocaine associated priapism". J. Urol., 161: 1817, 1999.

7. WEINER, D.M.; LOWE F.C.: "Surgical management of ischemic penile gangrene in diabetics with end stage atherosclerosis". J. Urol., 155: 926, 1996.

8. SEOANE VELA, M.A.; TERAN HINOJOSA, M.; PRESNO BERNAL, M. y cols.: "Calcifilaxis de Pene". Revista Mexicana de Urología, 64: 89, 2004.

9. ATALAY, A.C.; KARAMAN, M.I.: "Distal penile Gangrene with chronic renal failure". Int. J. Urol., 4: 431, 1997.

10. HATAMA, S.; NAKAMOTO, M.; IMAMURA, A. y cols.: "Penile gangrene in patient with diabetic nephropathy on continuous ambulatory peritoneal dialysis". Nipp. Jin. Gakk Shi Japanese Journal of Nephrology, 33: 863, 1991.

11. JACOBSOHN, H.A.; JENKINS, P.G.; JACOBSOHN, K.M.: "Penile calciphylaxis". Urol., 60: 344, 2002.

12. STEIN, M.; ANDERSON, C.; RICCIARDI, R. y cols.: "Penile gangrene associated with chronic renal failure: report of 7 cases and review of the literature". J. Urol., 152: 2014, 1994.

13. BARTHELMES, L.; CHEZHIAN, C.; THOMAS, K.J.: "Progression to wet gangrene in penile necrosis and calciphylaxis". Int. Urol. Nephrol., 34: 231, 2002.

14. KARPMAN, E.; DAS, S.; KURZROCK, E.A.: "Penile calciphylaxis: Analysis of risk factors and mortality". J. Urol., 169: 2206, 2003. 